\title{
Periodically Estimated Reflection Coefficient Measurement Uncertainties for a Vector Network Analyzer
}

\author{
Leonard E. Duda \\ Primary Electrical Standards Department \\ Sandia National Laboratories \\ Albuquerque, NM 87185 \\ (505)-844-3304 \\ leduda@sandia.gov
}

\begin{abstract}
This paper describes the model and method used to obtain the periodically estimated uncertainties for measurement of the scattering parameters $S_{11}$ and $S_{22}$ on a Vector Network Analyzer (VNA). A thru-reflect-line (TRL) method is employed as a second tier calibration to obtain uncertainty estimates using an NIST-calibrated standard. An example of tabulated listings of these uncertainty estimates is presented and the uncertainties obtained for a VNA with $7 \mathrm{~mm}$, $3.5 \mathrm{~mm}$, and type $\mathrm{N}$ coaxial interfaces used in our laboratory over several years are summarized.
\end{abstract}

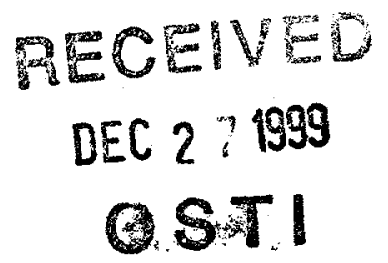

\section{INTRODUCTION}

Vector Network Analyzers (VNA) are widely used to measure the scattering parameters of a variety of microwave devices. Their usefulness lies in their broadband capability, ease of use, and high accuracy when properly "calibrated." There are, however, two issues that users of a VNA must consider. The first issue is whether the VNA is performing properly and therefore making accurate measurements while the second issue is the uncertainty or the error limits assigned to those measurements. The first issue has to do with the adequacy of the VNA "calibration" and the proper operation of all components in the system. The VNA operation and proper "calibration" can be verified with check standards or verification kits. The second issue is more complex. One option is to simply quote the VNA uncertainty specifications from the manufacturer for the measurements. However, this approach only gives typical uncertainties for the VNA; an individual VNA may have larger uncertainties than these typical uncertainties. Also, using the manufacturer's specifications usually does not account for the unique conditions (power level, connector type, for example) used for the VNA measurements. Another option is to assign type A or B uncertainties to all the individual components of the VNA and then to combine all of these uncertainty estimates into a final uncertainty value. This approach is difficult, time consuming, and may not be possible (except at the factory) because of unknown performance characteristics of certain VNA components or interactions between components. In addition, neither of these approaches provides uncertainties having a direct path traceable to national standards.

Because of the above considerations, the Primary Standards Laboratory at Sandia National Laboratories has developed another approach that is based on analyses of the measured performance of the VNA using traceable standards [1]. With these measurements and appropriate models, it is possible to develop uncertainties that apply directly to a specific VNA system.

Sandia is a multiprogram laboratory operated by Sandia Corporation, a Lockheed Martin Company, for the United States Department of Energy under contract DE-AC04-94AL85000. 
The process to calculate estimated uncertainties for a Vector Network Analyzer (VNA) requires setting the VNA to some desired conditions that includes a frequency set, power level, averaging factor, etc. With these conditions set, the VNA is "calibrated" using the appropriate calibration kit for the test port connectors. After the VNA is "calibrated," a series of measurements on an NIST-certified air line, and some standard devices, is performed as the first step in the uncertainty analysis. For the uncertainty analysis, each type of connector is treated separately. This means that there is a unique set of calculated uncertainties for APC-7 mm, type N, APC-3.5 $\mathrm{mm}$, etc.

In this paper, the analysis method, employing the TRL technique, used to calculate the residual uncertainties of a microwave network analyzer applicable over the frequency range of $25 \mathrm{MHz}$ to $50 \mathrm{GHz}$ (depending on the capability of the VNA) is briefly described. This method utilizes measurements on NIST-certified air lines plus additional measurements to estimate the combined standard uncertainties for measurements using the network analyzer. The uncertainties of the NIST standards are incorporated into the analysis by means of a Monte Carlo technique. Also included in the analysis is the repeatability of the measurement process. The final uncertainties assigned to a network analyzer then provide the basis for estimating the uncertainties assigned to measured values of specific devices. Finally, the paper summarizes the typical results of this method for characterizing network analyzer uncertainties for several connector types as obtained in our laboratory over a several year period.

\section{THEORY}

The Thru-Reflect-Line (TRL) technique offers an attractive way to determine the parameters of two error boxes at the test ports of a VNA. Because this technique uses an air line standard, it has a lower frequency limit of approximately 25 to $50 \mathrm{MHz}$. Conceptually, the VNA is viewed as shown in Figure 1. The conceptual model, illustrated in Figure 1, shows that when a deviceunder-test (DUT) with a true reflection coefficient of $\Gamma_{1}$ is connected to Test Port 1 , the VNA will produce a reading of $\Gamma_{1}^{m}$. This reading is the result of the corrupting influence of Error Box $\mathrm{X}$. In terms of the error box scattering parameters, $S_{i j}^{X}$ (which are complex quantities), the reflection coefficient, $\Gamma_{1}^{m}$, is given by

$$
\Gamma_{1}^{m}=S_{11}^{X}+\frac{S_{12}^{X} S_{21}^{X} \Gamma_{1}}{1-S_{22}^{X} \Gamma_{1}}=\frac{\left(S_{12}^{X} S_{21}^{X}-S_{11}^{X} S_{22}^{X}\right) \Gamma_{1}+S_{11}^{X}}{-S_{22}^{X} \Gamma_{1}+1}=\frac{a_{X} \Gamma_{1}+b_{X}}{c_{X} \Gamma_{1}+1}
$$

The right side of Eqn. 1 displays the equation in terms of the bi-linear or linear fractional transformation [2]. In order to determine the corrupting effects of the Error Box X, the values of the complex parameters $a_{X}, b_{X}$, and $c_{X}$ must be determined from measurements of the scattering parameters. The equivalent expression for Error Box $\mathrm{Y}$ is

$$
\Gamma_{2}^{m}=S_{22}^{Y}+\frac{S_{12}^{Y} S_{21}^{Y} \Gamma_{2}}{1-S_{11}^{Y} \Gamma_{2}}=\frac{\left(S_{12}^{Y} S_{21}^{Y}-S_{11}^{Y} S_{22}^{Y}\right) \Gamma_{2}+S_{22}^{Y}}{-S_{11}^{Y} \Gamma_{2}+1}=\frac{a_{Y} \Gamma_{2}+b_{Y}}{c_{Y} \Gamma_{2}+1}
$$




\section{DISCLAIMER}

This report was prepared as an account of work sponsored by an agency of the United States Government. Neither the United States Government nor any agency thereof, nor any of their employees, make any warranty, express or implied, or assumes any legal liability or responsibility for the accuracy, completeness, or usefulness of any information, apparatus, product, or process disclosed, or represents that its use would not infringe privately owned rights. Reference herein to any specific commercial product, process, or service by trade name, trademark, manufacturer, or otherwise does not necessarily constitute or imply its endorsement, recommendation, or favoring by the United States Government or any agency thereof. The views and opinions of authors expressed herein do not necessarily state or reflect those of the United States Government or any agency thereof. 


\section{DISCLAIMER}

Portions of this document may be illegible in electronic image products. Images are produced from the best available original document. 


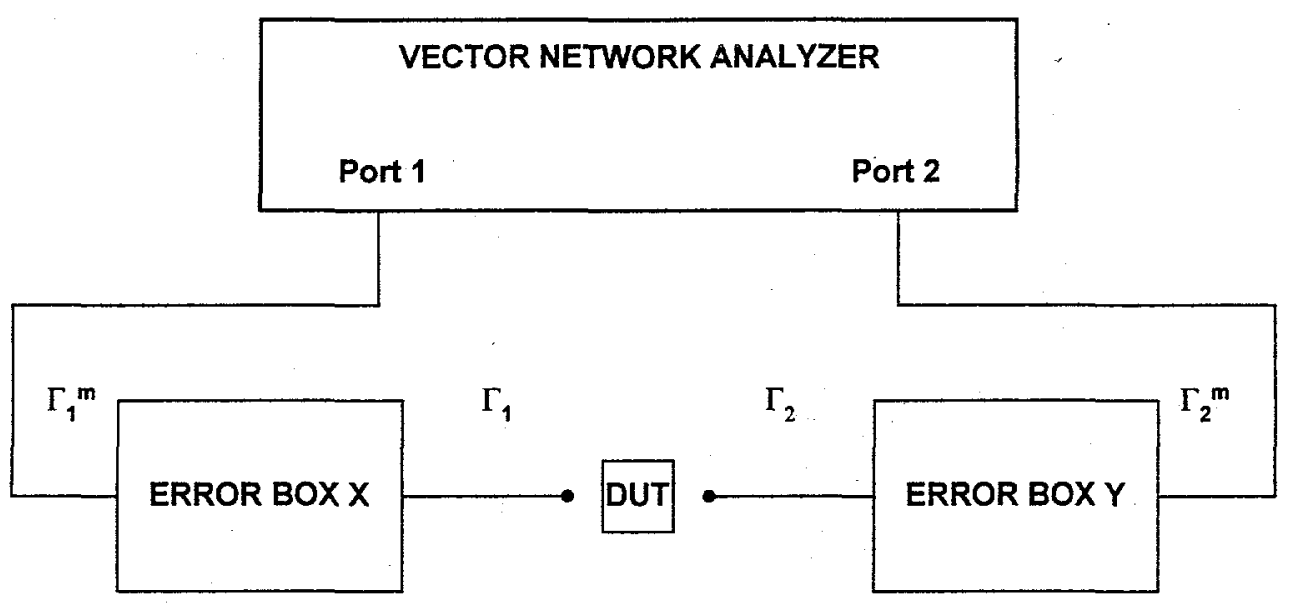

Figure 1. Conceptual diagram of a VNA and the error boxes at each port.

The TRL technique provides an efficient method to obtain the required measurements while requiring only one standard - the complex characteristic impedance, $Z_{l}$, of a transmission line. The advantage of the TRL method is that only one certified complex value is required and that the uncertainty on this quantity is very small for an air dielectric coaxial line (air line). It therefore follows that the residual uncertainties of the VNA may be very well characterized.

In order to obtain the error box parameters $a_{X}, b_{X}, c_{X}, a_{Y}, b_{Y}$, and $c_{Y}$, measurements of a thru line, an air line, an uncalibrated open, and an uncalibrated short are made at the test ports of the VNA. Using the measurements, Eqns. 1 and 2 are solved for the error box terms. Next, it is necessary to account for the fact that the true reflection coefficient is relative to impedance $Z_{i}$. This is accomplished by relating the true reflection coefficient computed from the measurements, $\Gamma_{1}$, to the true reflection coefficient in a $50 \mathrm{ohm}$ system, ${ }^{50} \Gamma_{1}$. Eqn. 1 may be inverted to yield

$$
\Gamma_{1}=\frac{\Gamma_{1}^{m}-b_{X}}{a_{X}-c_{X} \Gamma_{1}^{m}} .
$$

The true reflection coefficient relative to $Z_{l}$ may be related to ${ }^{50} \Gamma_{1}$ by

$$
\Gamma_{1}=\frac{{ }^{50} \Gamma_{1}\left(50+Z_{l}\right)+\left(50-Z_{l}\right)}{{ }^{50} \Gamma_{1}\left(50-Z_{l}\right)+\left(50+Z_{l}\right)} .
$$

Substituting Eqn. 4 into Eqn. 3 and rearranging gives

$$
{ }^{s 0} \Gamma_{1}=\frac{\Gamma_{1}^{m}-B}{A-C \Gamma_{1}^{m}},
$$


where

$$
A=\frac{a_{X}\left(50+Z_{l}\right)+b_{X}\left(50-Z_{l}\right)}{c_{X}\left(50-Z_{l}\right)+\left(50+Z_{l}\right)} \quad B=\frac{a_{X}\left(50-Z_{l}\right)+b_{X}\left(50+Z_{l}\right)}{c_{X}\left(50-Z_{l}\right)+\left(50+Z_{l}\right)} C=\frac{c_{X}\left(50+Z_{l}\right)+\left(50-Z_{l}\right)}{c_{X}\left(50-Z_{l}\right)+\left(50+Z_{l}\right)} .(6)
$$

Therefore, Eqn. 5 is used to estimate the value of ${ }^{50} \Gamma_{1}$ and then to compute the errors in the measurement.

To include the uncertainties in the standards (NIST certified air lines), a Monte Carlo technique [3] is utilized. In this technique, a normal distribution is assumed for the uncertainties of the standards. Using the Box-Muller transformation [3], a value is selected for the standard. This value is then used in the computation of the error box terms. The mean value and standard deviation of 101 computations are calculated for the errors which are given by

$$
\begin{aligned}
& E_{\text {mag }}=\left\|\Gamma_{\text {true }}|-| \Gamma_{\text {calc }}\right\| \\
& E_{\text {angle }}=\operatorname{Arg}\left(\Gamma_{\text {true }}-\Gamma_{\text {caic }}\right)
\end{aligned}
$$

where $\Gamma_{\text {true }}$ is the expected value of the reflection coefficient and $\Gamma_{\text {calc }}$ is the calculated value. $E_{\text {mag }}$ and $E_{\text {angle }}$ are the errors in the reflection coefficient magnitude and phase angle, respectively. The standard deviations of $E_{\text {mag }}$ and $E_{\text {angle }}$ are calculated to be Type A standard uncertainties [4]. The final uncertainties include a coverage factor which is usually $k=3[5]$.

An example of the results of the analysis and resulting uncertainty calculation for APC-7 mm connectors is shown in Table 1. The table shows both the errors and uncertainties (three standard deviations) of the model analysis for fourteen reflection coefficient values ranging from 0.003 to 1 as a function of frequency. Only part of the complete frequency set is shown in the table. The process which results in the uncertainty estimates for a particular VNA (i.e. including calibration kit for the specific connector type, averaging factor, power level, etc.) is called "certification" so as to distinguish it from the VNA "calibration" process. The VNA that has undergone this treatment is referred to as "certified." The entire process of setup, data acquisition, calculation, and even certificate printing is controlled by computer software [6].

As shown in the table, the method computes estimated uncertainties for both the magnitude and the argument of the phase angle of $S_{11}$ and $S_{22}$. The value in the error column should be small; usually much less than the estimated uncertainty. If it is not small, a potential problem with the measurement is indicated. The problem could arise from a variety of sources including improper VNA "calibration," calibration kit device failure, VNA hardware failure (e.g. the internal components within the test set), or improper connection of the measurement standards. 
Table 1. Calculated VNA uncertainties for APC-7 mm connectors. The uncertainties calculated follow the guidelines from ANSI/NCSL Z540-2-1997 [11]. The error values quantify the validity of bias-free assumptions required by ANSI/NCSL Z540-2-1997 guidelines. The absolute value or magnitude of the reflection coefficient, $\Gamma$, is $\rho$ and designated by $R$ ho in the table.

$7 \mathrm{~mm}|\mathrm{~S} 11|$ Errors \& Expanded $(k=3.0)$ Uncertainties

\begin{tabular}{cccccccccc}
\hline $\begin{array}{c}\text { Measured } \\
\text { Rho }\end{array}$ & \multicolumn{2}{c}{50.0} & \multicolumn{9}{c}{100.0} & \multicolumn{2}{c}{250.0} & \multicolumn{2}{c}{500.0} \\
& Error & Unc. & Error & Unc. & Error & Unc. & Error & Unc. \\
\hline .0030 & -.0004 & .0040 & -.0003 & .0037 & -.0004 & .0039 & -.0002 & .0030 \\
.0100 & -.0001 & .0042 & -.0001 & .0039 & -.0001 & .0041 & -.0001 & .0031 \\
.0150 & -.0001 & .0042 & -.0001 & .0039 & -.0001 & .0041 & -.0001 & .0031 \\
.0200 & -.0001 & .0042 & -.0001 & .0039 & -.0001 & .0041 & -.0001 & .0031 \\
.0300 & -.0001 & .0042 & -.0001 & .0039 & -.0001 & .0041 & -.0001 & .0031 \\
.0500 & -.0001 & .0042 & -.0001 & .0039 & -.0000 & .0041 & -.0001 & .0031 \\
.0700 & -.0001 & .0042 & -.0001 & .0039 & -.0000 & .0041 & -.0001 & .0031 \\
.1000 & -.0000 & .0042 & -.0001 & .0039 & -.0000 & .0041 & -.0001 & .0031 \\
.1500 & -.0000 & .0042 & -.0001 & .0039 & -.0000 & .0041 & -.0001 & .0031 \\
.2000 & -.0000 & .0041 & -.0001 & .0038 & 0.0000 & .0040 & -.0001 & .0030 \\
.3000 & -.0000 & .0041 & -.0002 & .0037 & 0.0000 & .0039 & -.0002 & .0029 \\
.4000 & -.0000 & .0040 & -.0002 & .0036 & .0001 & .0037 & -.0002 & .0028 \\
.5000 & -.0000 & .0039 & -.0002 & .0034 & .0001 & .0035 & -.0002 & .0026 \\
.9999 & 0.0000 & .0046 & -.0004 & .0035 & .0002 & .0028 & -.0004 & .0026 \\
\hline
\end{tabular}

$7 \mathrm{~mm}$ Arg (S11) Errors \& Expanded $(k=3.0)$ Uncertainties

\begin{tabular}{rrrrrrrrr}
\hline .0030 & 12.89 & 180.00 & .54 & 180.00 & .39 & 180.00 &. .43 & 180.00 \\
.0100 & .24 & 24.37 & .28 & 22.41 & .19 & 23.58 & .19 & 17.89 \\
.0150 & .19 & 16.16 & .23 & 14.87 & .15 & 15.64 & .15 & 11.89 \\
.0200 & .15 & 12.10 & .21 & 11.13 & .13 & 11.71 & .13 & 8.91 \\
.0300 & .12 & 8.06 & .18 & 7.42 & .11 & 7.80 & .10 & 5.94 \\
.0500 & .10 & 4.84 & .16 & 4.45 & .09 & 4.68 & .09 & 3.57 \\
.0700 & .08 & 3.46 & .15 & 3.19 & .08 & 3.35 & .08 & 2.55 \\
.1000 & .08 & 2.43 & .14 & 2.24 & .07 & 2.35 & .07 & 1.79 \\
.1500 & .07 & 1.63 & .14 & 1.50 & .07 & 1.58 & .07 & 1.21 \\
.2000 & .06 & 1.23 & .13 & 1.14 & .07 & 1.20 & .06 & .92 \\
.3000 & .06 & .84 & .13 & .78 & .06 & .83 & .06 & .63 \\
.4000 & .06 & .65 & .13 & .61 & .06 & .65 & .06 & .50 \\
.5000 & .06 & .55 & .13 & .52 & .06 & .55 & .06 & .42 \\
.9999 & .06 & .41 & .13 & .40 & .06 & .41 & .06 & .33 \\
\hline
\end{tabular}

The uncertainties calculated by these methods are included as Type A errors when measuring a device with the "calibrated" network analyzer. The expanded uncertainty that is assigned to a device when it is measured on a calibrated network analyzer is

$$
U_{\exp }=k \cdot \sqrt{U_{m}^{2}+U_{v}^{2}}
$$

where $U_{\text {exp }}$ is the expanded uncertainty, $\mathrm{k}$ is the coverage factor, $U_{m}$ is the Type A measurement uncertainty or repeatability observed when the device is measured, and $U_{v}$ is the VNA uncertainty calculated from the method described in this paper. Proper network analyzer 
calibration, multiple measurement on a DUT, and including the estimate of the residual VNA errors described here provide a plausible estimate of the final DUT measurement uncertainty.

\section{RESULTS}

In this section the uncertainty estimates for a VNA system with $7 \mathrm{~mm}, 3.5 \mathrm{~mm}$, and type $\mathrm{N}$ coaxial interfaces are summarized and discussed. Four data sets for each connector obtained over four years were analyzed. These four data sets represent annual certification measurements on the network analyzer. The 7-mm connector used the TRL calibration kit while the other connectors employed the SOLT [short, open, load (with a sliding load), thru] calibration kit. Thirteen measurement frequencies of 50,100,250, 500,1500, 2500, 4500, 5500, 7500, 9500, 12500, 15500 , and $17500 \mathrm{MHz}$ were used for certification of the VNA for each connector. The mean uncertainty and standard deviation of the mean for the uncertainty estimate at each frequency from the four data sets were calculated. Only the magnitude of the reflection coefficient $(\rho)$ is examined in this paper.

Figure 2 shows the plot of the mean uncertainty values for six $\rho$ values of $0.010,0.050,0.100$, $0.300,0.500$, and 1.0 for the $S_{11}$ measurement. Except for $\rho=1.0$, the magnitude of the uncertainties are similar. Note that there is clearly a frequency dependence with a general trend of increasing uncertainty with increasing frequency (except for frequencies below $2000 \mathrm{MHz}$ ). Figure 3 shows the standard deviation of the mean for the data from Figure 2. Figure 4 plots the relative error or relative uncertainty for these calculations $[7,8,9]$. The relative error is lower at frequencies above $2000 \mathrm{MHz}$. Note that the mean uncertainties values are quite small so that any variation, denoted by the standard deviation, results in comparatively large relative errors.

To further understand the variation in the uncertainty values, as shown by the standard deviation plot in Figure 3, the last certification measurement data set for the 7-mm connector was reanalyzed six times and saved. These data were analyzed to observe the effect of the Monte Carlo technique on the results. It was found that the mean uncertainty values obtained were very similar to those shown in Figure 2. However, the standard deviation and the relative error were reduced by a factor of greater than 100 . This fact demonstrates that the variation seen in the data shown by the standard deviation or the relative error plots is primarily the result of changes occurring in the system between certifications and is not the result of the calculational method.

Figures 5 and 6 plot the mean uncertainty values and relative errors for the $3.5-\mathrm{mm}$ connector. These results show the same general trend as the $7-\mathrm{mm}$ connector results. However, the uncertainty values are larger than the 7-mm uncertainty values. Part of this increase may be attributed to the larger relative uncertainty of the standard air line used for the $3.5 \mathrm{~mm}$ measurements compared to the $7 \mathrm{~mm}$ measurements. A significant difference between these two sets of data is evident at higher frequencies. The $3.5-\mathrm{mm}$ uncertainties at the higher frequencies are smaller than the values at lower frequencies. The opposite is true for the $7-\mathrm{mm}$ connector data. 


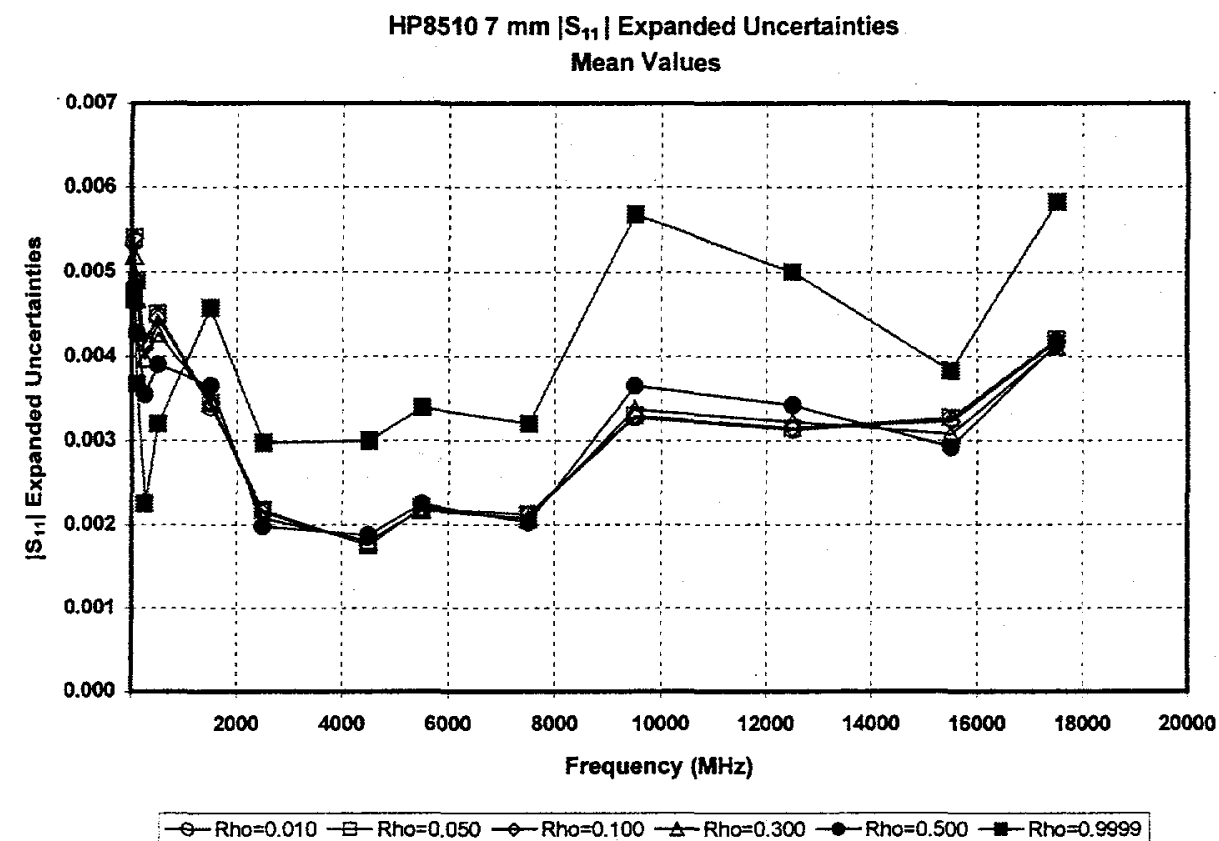

Figure 2. Mean values of calculated VNA uncertainties for the 7-mm connector from four data sets measured over a four-year period [10].

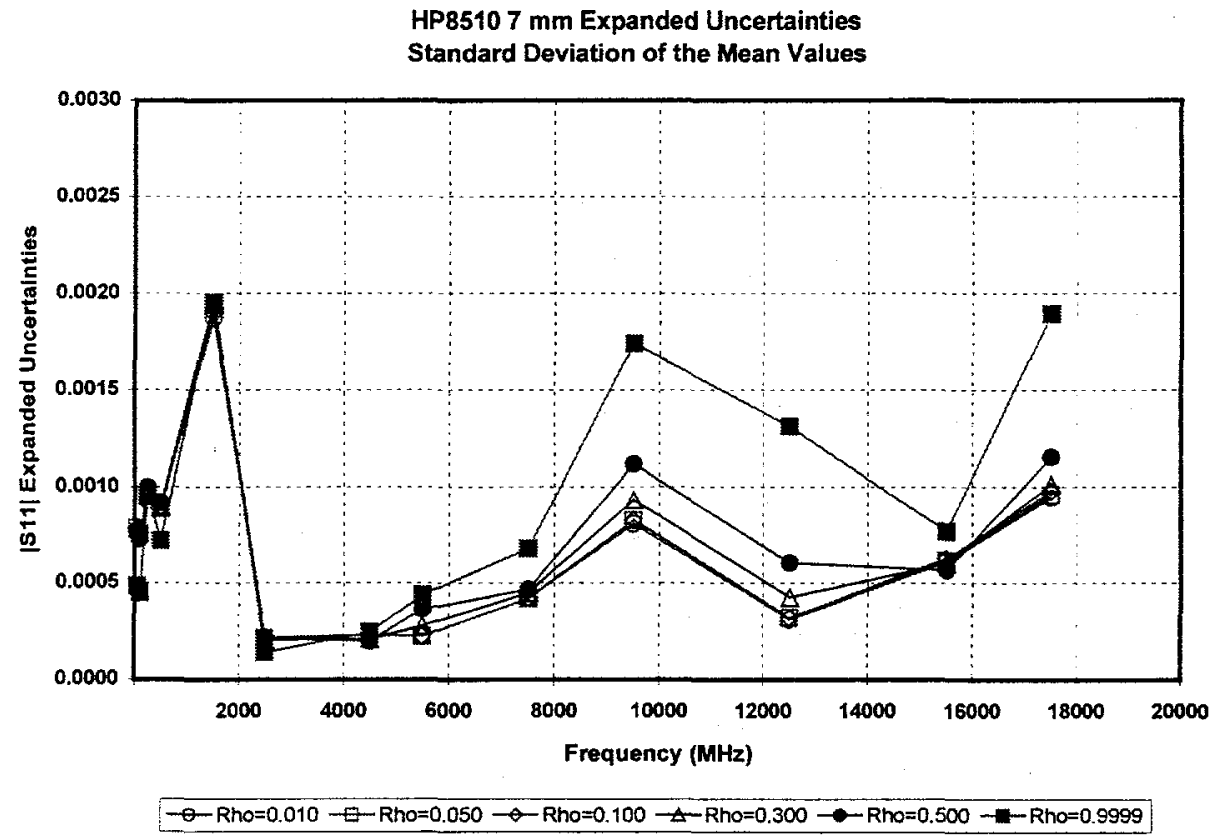

Figure 3. Standard deviation of the mean values of calculated VNA uncertainties for the 7-mm connector from four data sets measured over a four-year period. 


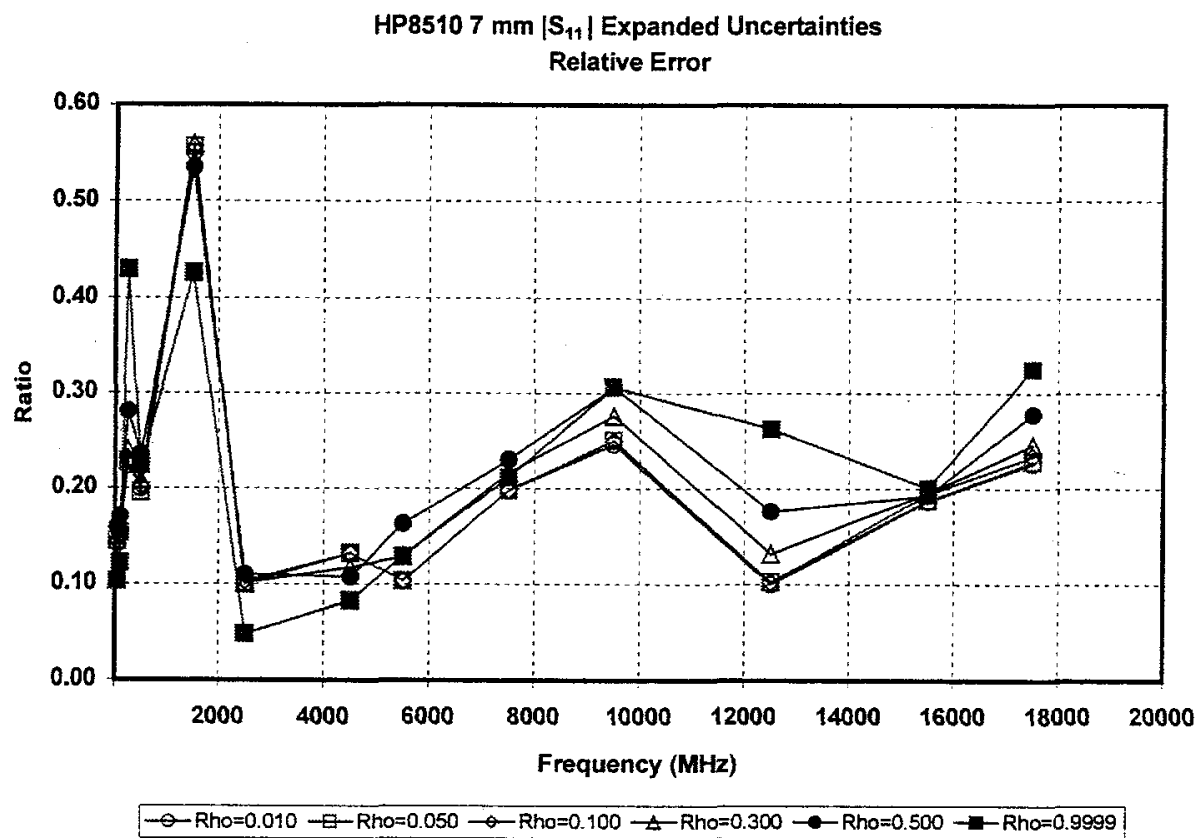

Figure 4. Relative error of calculated VNA uncertainties for the 7-mm connector from four data sets measured over a four-year period. The relative error is the ratio of the standard deviation to the mean value.

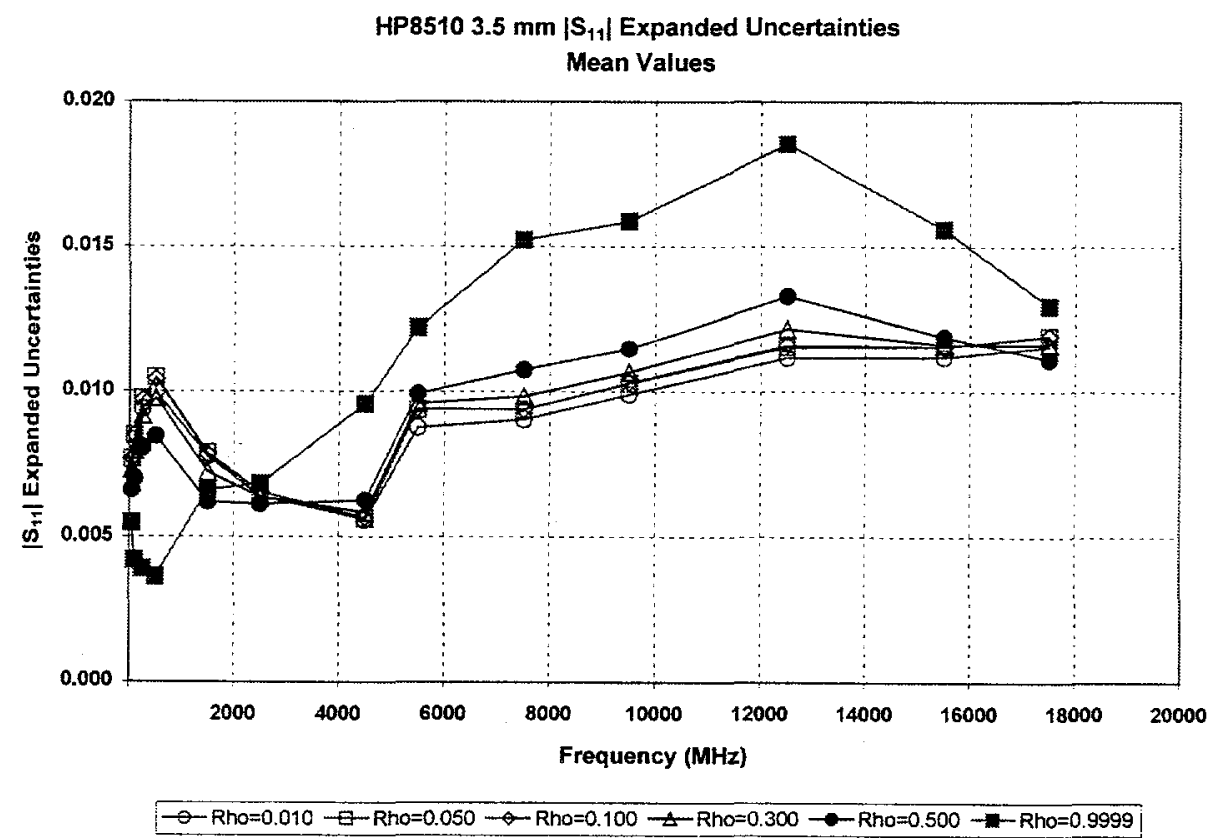

Figure 5. Mean values of calculated VNA uncertainties for the $3.5-\mathrm{mm}$ connector from four data sets measured over a four-year period. 


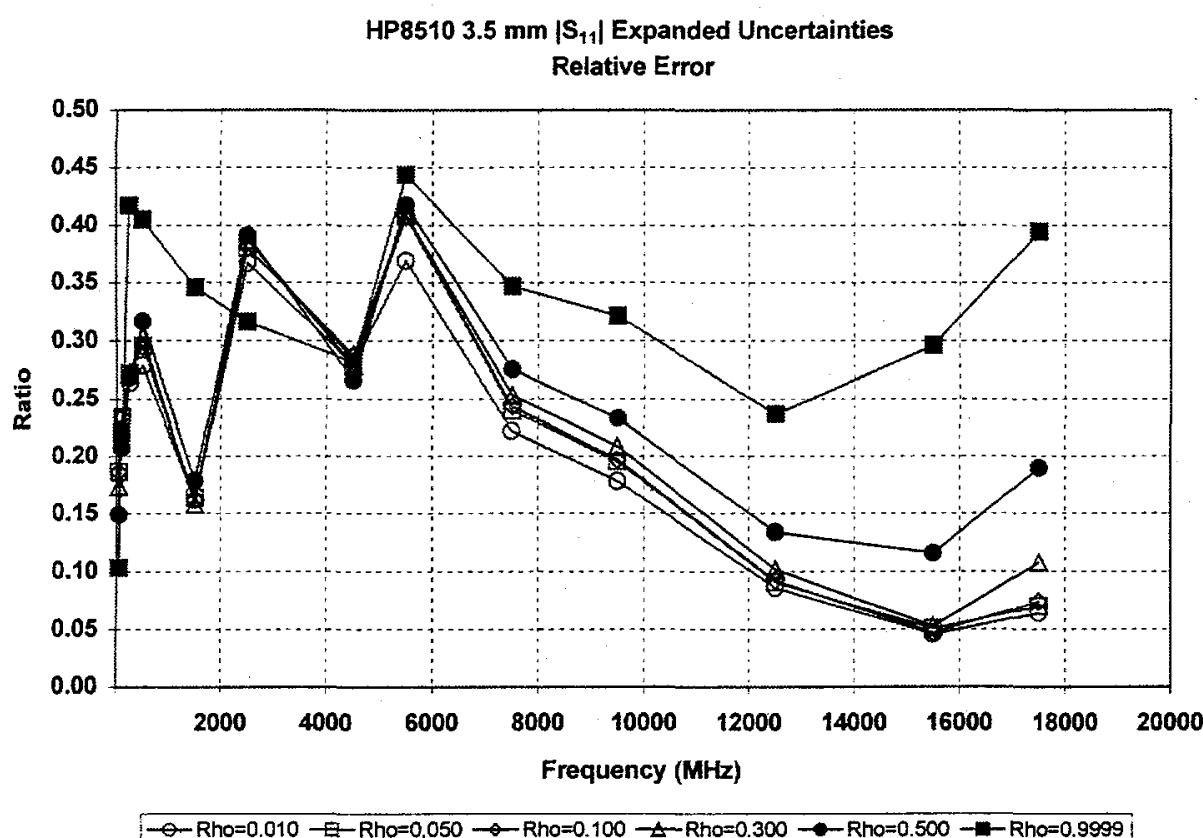

Figure 6. Relative error of calculated VNA uncertainties for the 3.5-mm connector from four data sets measured over a four-year period. The relative error is the ratio of the standard deviation to the mean value.

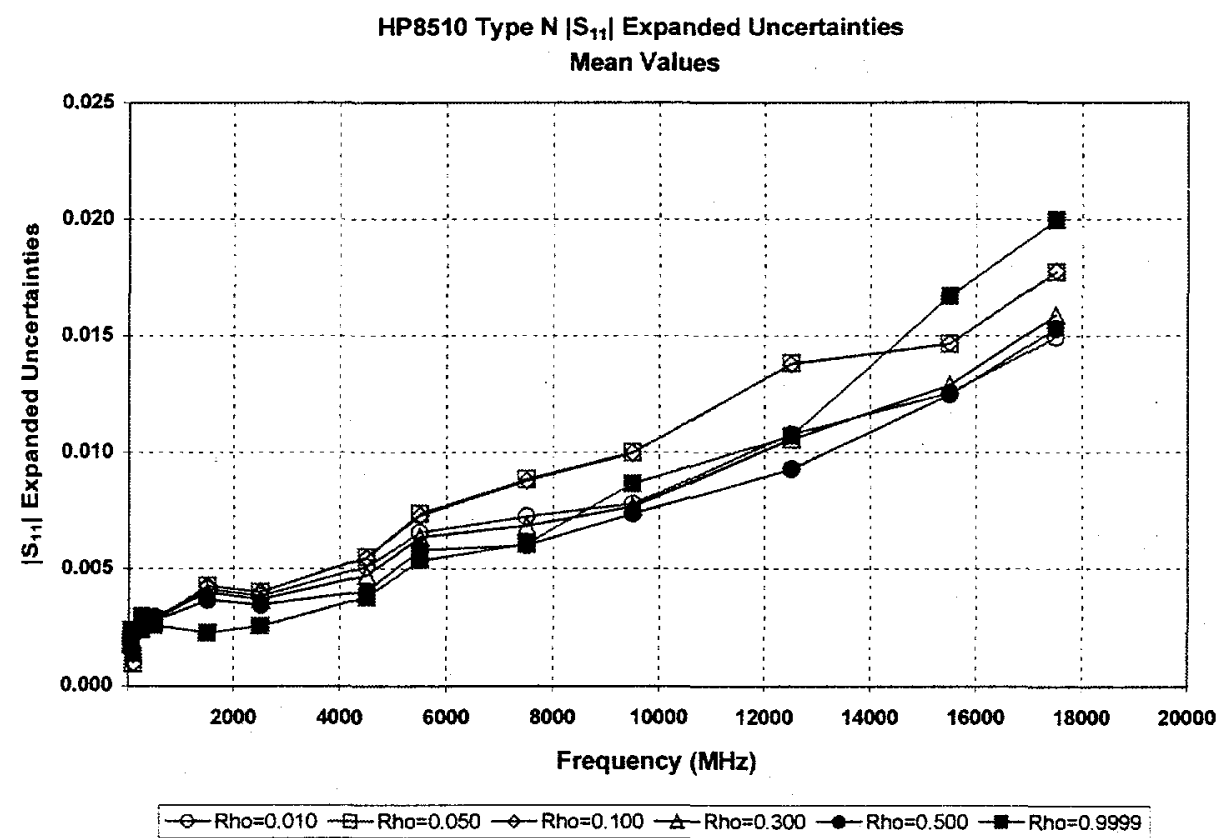

Figure 7. Mean values of calculated VNA uncertainties for the type $\mathrm{N}$ connector from four data sets measured over a four-year period. 


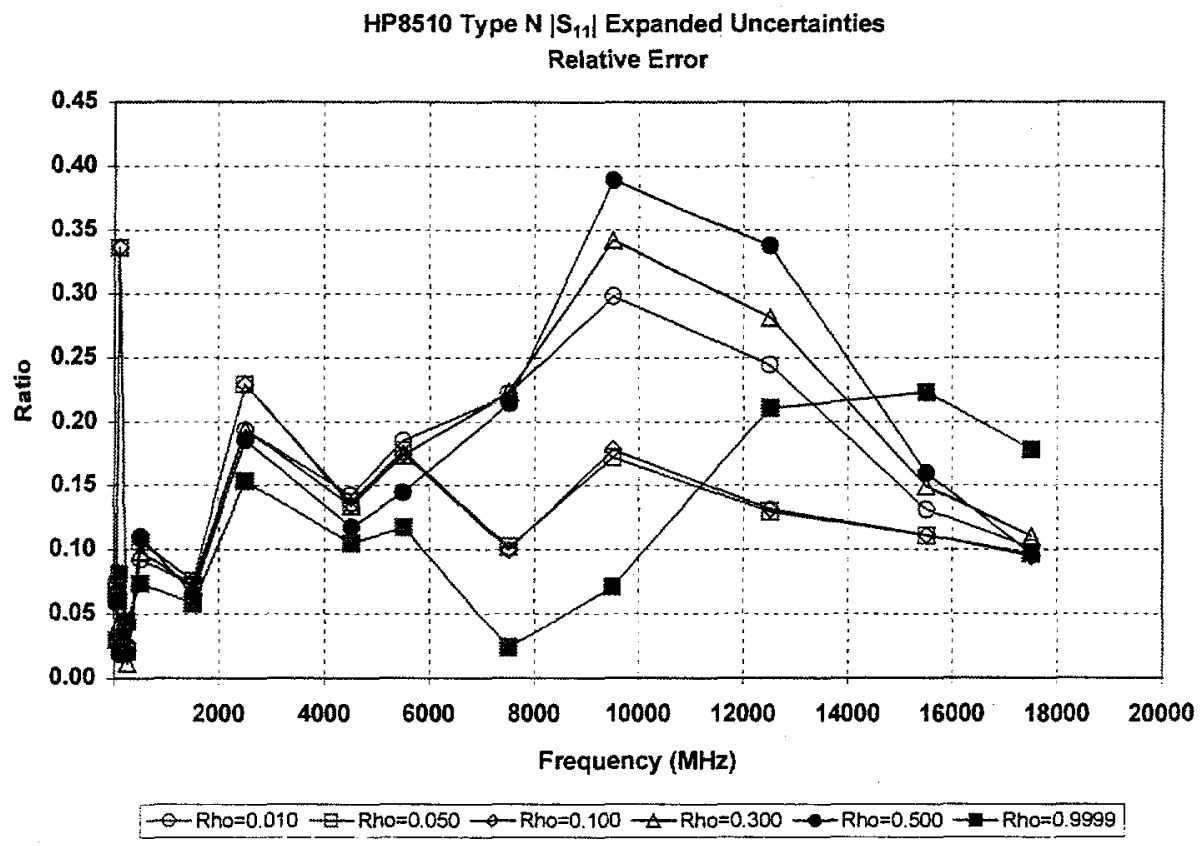

Figure 8. Relative error of calculated VNA uncertainties for the type $\mathrm{N}$ connector from four data sets measured over a four-year period. The relative error is the ratio of the standard deviation to the mean value.

Figures 7 and 8 plot the mean uncertainty values and relative errors for the type $\mathrm{N}$ connector. The type $\mathrm{N}$ results clearly show a very pronounced increase in uncertainty value with increasing frequency. Unlike the other two connector results, the $\rho=1.0$ data do not produce the largest uncertainty values. In fact, all $\rho$ values for type $\mathrm{N}$ exhibit much less scatter than the other connectors. The relative error for type $\mathrm{N}$ is largest in the middle frequency range.

The uncertainty estimates for $\left|\mathrm{S}_{22}\right|, \operatorname{Unc}\left(\left|\mathrm{S}_{22}\right|\right)$, show general trends for each connector similar to the trends shown above for $\left|\mathrm{S}_{11}\right|$. However, there is still a difference between the two VNA ports that is illustrated in Figure 9 for the 7 -mm connector. Figure 9 plots the quantity (Unc $\left|\mathrm{S}_{11}\right|-$ $\left.\operatorname{Unc}\left(\left|\mathrm{S}_{22}\right|\right)\right) / \operatorname{Unc}\left(\left|\mathrm{S}_{11}\right|\right)$, where Unc( ) denotes the uncertainty of the listed s-parameter, as a function of frequency. The plot shows that, except for the case of $\rho=1.0$, all other $\rho$ values display a substantial difference between the two scattering parameters. However, again it must be noted that the observed differences are generally differences between small numbers. For example, Table 2 shows the mean uncertainties at a frequency of $7500 \mathrm{MHz}$ for the two scattering parameters. The uncertainties are quite small and this causes their difference to be magnified. 


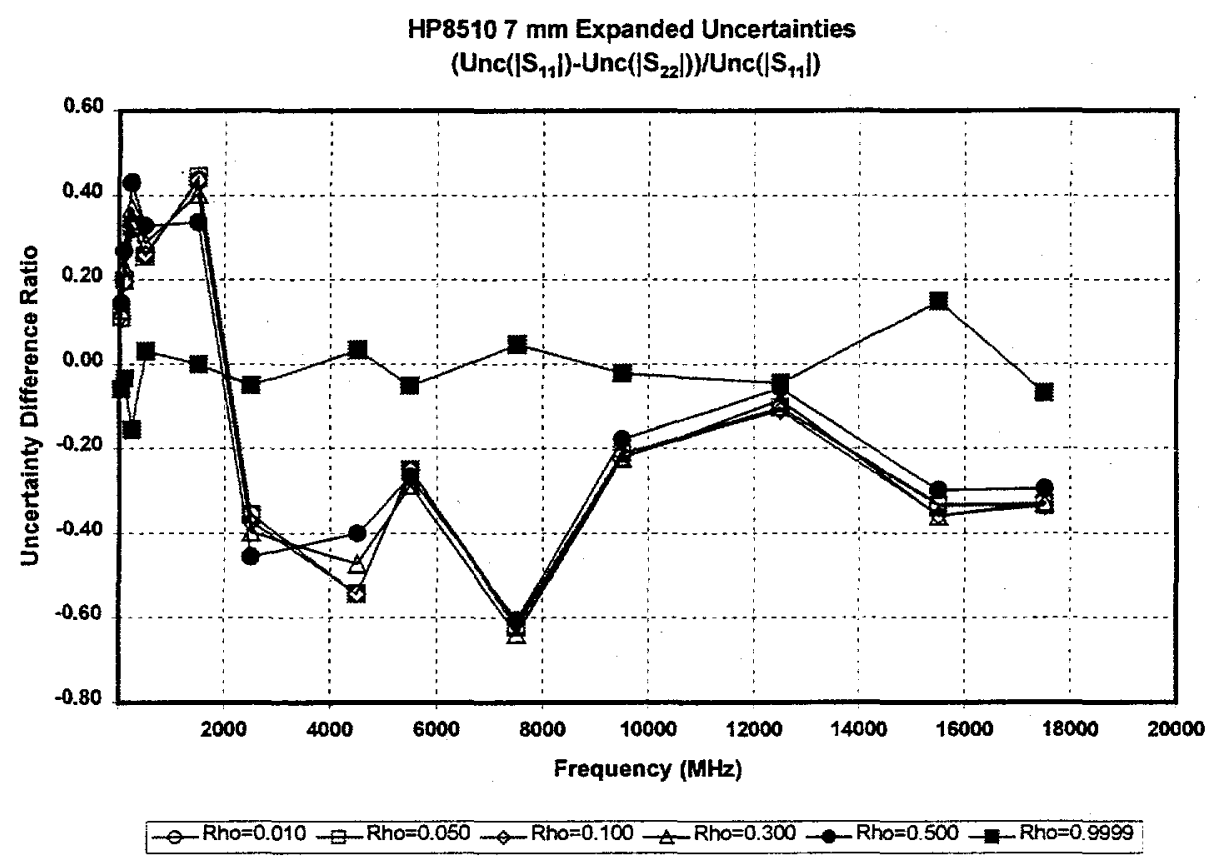

Figure 9. Difference between the uncertainty magnitude of the two scattering parameters $S_{11}$ and $S_{22}$ relative to the uncertainty magnitude of $S_{11}$. The data are the mean values from the four data sets for the VNA uncertainties for the 7-mm connector.

Table 2. Comparison between the $\left|S_{11}\right|$ and $\left|S_{22}\right|$ mean uncertainties at $7500 \mathrm{MHz}$ for the 7 -mm connector. The first column labeled $\rho$ represents the measured value of the reflection coefficient magnitude. The uncertainty remains fairly constant with $\rho$ which means that the relative uncertainty tends to decrease as $\rho$ increases.

\begin{tabular}{c|c|c}
\hline$\rho$ & $\left|\mathbf{S}_{11}\right|$ & {$\left[\mathbf{S}_{22} \mid\right.$} \\
\hline 0.010 & 0.0021 & 0.0035 \\
0.050 & 0.0021 & 0.0035 \\
0.100 & 0.0021 & 0.0034 \\
0.300 & 0.0021 & 0.0034 \\
0.500 & 0.0020 & 0.0033 \\
1.000 & 0.0032 & 0.0031 \\
\hline
\end{tabular}

\section{SUMMARY}

This paper has presented a method to perform a second-tier calibration of a VNA (called a "certification") that produces uncertainty estimates for a specific VNA under defined measurement conditions. These uncertainty estimates may be used to provide an expanded uncertainty for subsequent measurements with the VNA. The $\left|\mathrm{S}_{11}\right|$ results for $7 \mathrm{~mm}, 3.5 \mathrm{~mm}$, and type $\mathrm{N}$ connectors clearly show that the estimated uncertainties are dependent upon the particular 
configuration of the VNA. The $7 \mathrm{~mm}$ uncertainties are lower than the uncertainties from the other connectors. Both $3.5 \mathrm{~mm}$ and type $\mathrm{N}$ results show a similar uncertainty magnitude but the dependence on frequency is quite different for the two connectors. The two scattering parameters exhibit similar trends for a given connector but there are differences, sometimes substantial, between the uncertainties. The data presented here clearly show the usefulness of this method for providing an uncertainty estimate that depends explicity on the VNA system employed.

\section{REFERENCES}

[1] L. E. Duda, "Determination of the Uncertainties of Reflection Coefficient Measurements of a Microwave Network Analyzer," Proceedings of the 1998 NCSL Workshop and Symposium, Albuquerque, NM, July 19-23, 1998.

[2] G. F. Engen, Microwave Circuit Theory and Foundations of Microwave Metrology, Peter Peregrinus Ltd., London, UK, 1992.

[3] James V Beck and Kenneth J Arnold, Parameter Estimation in Engineering and Science, John Wiley and Sons, New York, 1977.

[4] Barry N. Taylor and Chris E. Kuyatt, "Guidelines for Evaluating and Expressing the Uncertainty of NIST Measurement Results," NIST Technical Note 1297, September 1994.

[5] The symbol, $\mathrm{k}$, used here denotes the coverage factor. It multiplies the combined standard uncertainty to obtain the expanded uncertainty.

[6] The software program used is named CERTVANA. It is an HP Basic program (that may be run under either HP Basic or HTBasic) that calculates the uncertainties for all the complex scattering parameters measured by the network analyzers. Besides the reflection coefficient $\left(\mathrm{S}_{11}\right.$ and $\left.\mathrm{S}_{22}\right)$, the program also calculates uncertainties for $\left|\mathrm{S}_{21}\right|,\left|\mathrm{S}_{12}\right|$, and $\operatorname{Arg}\left(\mathrm{S}_{21}\right)$, and $\operatorname{Arg}\left(\mathrm{S}_{12}\right)$. [7] D. C. Baird, Experimentation: An Introduction to Measurement Theory and Experiment Design, Prentice-Hall, Inc., Englewood Cliffs, New York, 1995.

[8] John R. Taylor, An Introduction to Error Analysis, University Science Books, Sausalito, California, 1997.

[9] The relative error or the relative uncertainty (sometimes called the fractional uncertainty or the precision) is the uncertainty divided by the measured value. Here the standard deviation of the mean divided by the mean is used.

[10] Reference to a commercial product is included for completeness only and implies neither endorsement by Sandia National Laboratories or the Department of Energy nor lack of a suitable substitute.

[11] ANSI/NCSL Z540-2-1997, "U.S. Guide to the Expression of Uncertainty in Measurement," National Conference of Standards Laboratories, October 1997. 